\title{
New discrete inequalities of Hermite-Hadamard type for convex functions
}

Pshtiwan Othman Mohammed ${ }^{1}$, Thabet Abdeljawad ${ }^{2,3,4^{*}}$ (D), Manar A. Alqudah ${ }^{*^{*}}$ and Fahd Jarad ${ }^{6}$

\section{"Correspondence:}

tabdeljawad@psu.edu.sa;

manarqudah@yahoo.com

${ }^{2}$ Department of Mathematics and

General Sciences, Prince Sultan

University, P.O. Box 66833, Riyadh

11586, Saudi Arabia

${ }^{5}$ Department of Mathematical

Sciences, Princess Nourah bint

Abdulrahman University, Riyadh,

Saudi Arabia

Full list of author information is

available at the end of the article

\begin{abstract}
We introduce new time scales on $\mathbb{Z}$. Based on this, we investigate the discrete inequality of Hermite-Hadamard type for discrete convex functions. Finally, we improve our result to investigate the discrete fractional inequality of Hermite-Hadamard type for the discrete convex functions involving the left nabla and right delta fractional sums.
\end{abstract}

MSC: 26D07; 26D10; 26D15; 26A33

Keywords: Time scale; Hermite-Hadamard inequality; Convex functions

\section{Introduction}

The integral inequality of Hermite-Hadamard type (or briefly HH-type) is a very interesting topic of mathematical analysis, this challenging topic has been developing very rapidly in the last three decades; see e.g. [1-5].

In the literature, there are two well-known types of $\mathrm{HH}$-type inequalities which were obtained by Sarikaya et al. in [6] and [7], respectively. Their results are, respectively,

$$
\Upsilon\left(\frac{\kappa_{1}+\kappa_{2}}{2}\right) \leq \frac{\Gamma(\varepsilon+1)}{2\left(\kappa_{2}-\kappa_{1}\right)}\left[{ }^{R L} I_{\kappa_{1+}+}^{\varepsilon} \Upsilon\left(\kappa_{2}\right)+{ }^{R L} I_{\kappa_{2}-}^{\varepsilon} \Upsilon\left(\kappa_{1}\right)\right] \leq \frac{\Upsilon\left(\kappa_{1}\right)+\Upsilon\left(\kappa_{2}\right)}{2}
$$

and

$$
\begin{aligned}
\Upsilon\left(\frac{\kappa_{1}+\kappa_{2}}{2}\right) & \leq \frac{2^{\varepsilon-1} \Gamma(\varepsilon+1)}{\left(\kappa_{2}-\kappa_{1}\right)^{\varepsilon}}\left[{ }^{R L} I_{\left(\frac{\kappa_{1}+\kappa_{2}}{2}\right)+}^{\varepsilon} \Upsilon\left(\kappa_{2}\right)+{ }^{R L} I_{\left(\frac{\kappa_{1}+\kappa_{2}}{2}\right)-}^{\varepsilon} \Upsilon\left(\kappa_{1}\right)\right] \\
& \leq \frac{\Upsilon\left(\kappa_{1}\right)+\Upsilon\left(\kappa_{2}\right)}{2} .
\end{aligned}
$$

These results are valid for any $\varepsilon>0$ and any $L^{1}$ convex function $\Upsilon:\left[\kappa_{1}, \kappa_{2}\right] \rightarrow \mathbb{R}$. The difference between (1.1) and (1.2) is that in (1.1) is that we are considering fractional integration from the two respective ends of the interval $\left[\kappa_{1}, \kappa_{2}\right]$ instead of from the center of the interval $\left[\kappa_{1}, \kappa_{2}\right]$ as used in (1.2).

(c) The Author(s) 2021. This article is licensed under a Creative Commons Attribution 4.0 International License, which permits use, sharing, adaptation, distribution and reproduction in any medium or format, as long as you give appropriate credit to the original author(s) and the source, provide a link to the Creative Commons licence, and indicate if changes were made. The images or other third party material in this article are included in the article's Creative Commons licence, unless indicated otherwise in a credit line to the material. If material is not included in the article's Creative Commons licence and your intended use is not permitted by statutory regulation or exceeds the permitted use, you will need to obtain permission directly from the copyright holder. To view a copy of this licence, visit http://creativecommons.org/licenses/by/4.0/. 
In the last ten years, the study of inequalities on time scales has received a lot of attention in the literature and has become important in both the fields of pure and of applied mathematics; see e.g. [8-13].

In 2016, Atici and Yildiz [10] obtained the discrete Hermite-Hadamard inequalities corresponding to (1.1) on the time scale $T_{\left[\kappa_{1}, \kappa_{2}\right]}:=\left\{h ; h=\frac{\kappa_{2}-c}{\kappa_{2}-\kappa_{1}}\right.$ for $\left.c \in\left[\kappa_{1}, \kappa_{2}\right]_{\mathbb{Z}}\right\}$, their results are as follows.

Theorem 1.1 Let $\Upsilon: \mathbb{Z} \rightarrow \mathbb{R}$ be a convex function on $\left[\kappa_{1}, \kappa_{2}\right]_{\mathbb{Z}}$ and $\kappa_{1}, \kappa_{2} \in \mathbb{Z}$ with $\kappa_{1}<\kappa_{2}$. If $\kappa_{1}+\kappa_{2}$ is an even number, then, for $\mathrm{c} \in \mathrm{T}_{\left[\kappa_{1}, \kappa_{2}\right]} \backslash\{0,1\}$, we have

$$
\Upsilon\left(\frac{\kappa_{1}+\kappa_{2}}{2}\right) \leq \frac{1}{2\left(\kappa_{2}-\kappa_{1}\right)}\left[\int_{\kappa_{1}}^{\kappa_{2}} \Upsilon(\mathrm{c}) \Delta \mathrm{c}+\int_{\kappa_{1}}^{\kappa_{2}} \Upsilon(\mathrm{c}) \nabla \mathrm{c}\right] \leq \frac{\Upsilon\left(\kappa_{1}\right)+\Upsilon\left(\kappa_{2}\right)}{2} .
$$

Theorem 1.2 Let $\Upsilon: \mathbb{Z} \rightarrow \mathbb{R}$ be a convex function on $\left[\kappa_{1}, \kappa_{2}\right]_{\mathbb{Z}}$ and $\kappa_{1}, \kappa_{2} \in \mathbb{Z}$ with $\kappa_{1}<\kappa_{2}$. If $\kappa_{1}+\kappa_{2}$ is an even number, then, for $\varepsilon>0$ and $\mathrm{c} \in \mathrm{T}_{\left[\kappa_{1}, \kappa_{2}\right]} \backslash\{0,1\}$, we have

$$
\begin{aligned}
\Upsilon\left(\frac{\kappa_{1}+\kappa_{2}}{2}\right) & \leq \frac{\Gamma(\varepsilon)}{2 \Omega\left(\kappa_{2}-\kappa_{1}\right)}\left[\left(\Delta_{\kappa_{2}-1}^{-\varepsilon} \Upsilon\right)(a-\varepsilon)+\left({ }_{\kappa_{1}+1} \nabla^{-\varepsilon} \Upsilon\right)\left(\kappa_{2}\right)\right] \\
& \leq \frac{\Upsilon\left(\kappa_{1}\right)+\Upsilon\left(\kappa_{2}\right)}{2},
\end{aligned}
$$

where

$$
\Omega=\int_{T_{\left[\kappa_{1}, \kappa_{2}\right]}}\left(\left(\kappa_{2}-\kappa_{1}\right) \mathrm{c}+\varepsilon-1\right)^{\overline{\varepsilon-1}} \hat{\Delta} \mathrm{c} .
$$

In the literature, the inequalities of HH-type are often connected with further integral inequalities which are called trapezoidal type (where the ends $a, b$ of the interval are used) or midpoint type (where the midpoint $\frac{\kappa_{1}+\kappa_{2}}{2}$ of the interval is used). Many inequalities of such a type have been obtained by many researchers; see e.g. [14-23].

In the present study, we obtain the new discrete inequalities of $\mathrm{HH}$-type corresponding to (1.2) on the new time scales $T_{\left[\frac{\kappa_{1}+\kappa_{2}}{2}, \kappa_{2}\right]}$, where

$$
\mathrm{T}_{\left[\frac{\kappa_{1}+\kappa_{2}}{2}, \kappa_{2}\right]}:=\left\{h ; h=\frac{2\left(\kappa_{2}-\mathrm{c}\right)}{\kappa_{2}-\kappa_{1}} \text { such that } \mathrm{c} \in\left[\frac{\kappa_{1}+\kappa_{2}}{2}, \kappa_{2}\right]_{\mathbb{Z}}\right\} \text {, }
$$

where $\left[\kappa_{1}, \kappa_{2}\right]_{\mathbb{Z}}=\left[\kappa_{1}, \kappa_{2}\right] \cap \mathbb{Z}$. We can observe that $\top_{\left[\frac{\kappa_{1}+\kappa_{2}}{2}, \kappa_{2}\right]}$ is a finite subset of the interval $[0,1]$.

\section{Discrete inequality of HH-type}

At first, we need to recall the following preliminary definitions and theorems of discrete time scales.

Definition 2.1 ([10]) Let $z_{1}, z_{2}$ be two elements of a time scale $\top$ with $z_{1}<z_{2}$. A function $\Upsilon: \top \rightarrow \mathbb{R}$ is said to be convex on $T$, if

$$
\Upsilon\left(\mathrm{c} z_{1}+(1-\mathrm{c}) z_{2}\right) \leq \mathrm{c} \Upsilon\left(z_{1}\right)+(1-\mathrm{c}) \Upsilon\left(z_{2}\right)
$$

holds for each $c \in T_{\left[z_{1}, z_{2}\right]}$. 
In the following theorem, we recall the time scale substitution rule.

Theorem 2.1 ([24]) Let $w: \mathbb{Z} \rightarrow \mathbb{R}$ be strictly increasing and $\hat{\top}:=w(\top)$ be a time scale. If $\Upsilon: \mathbb{Z} \rightarrow \mathbb{R}$ is an rd-continuous function and $w$ is differentiable with $r$-continuous derivative, then for $\kappa_{1}, \kappa_{2} \in T_{\left[\kappa_{1}, \kappa_{2}\right]}$ we have

$$
\int_{\kappa_{1}}^{\kappa_{2}} \Upsilon(\mathrm{c}) w^{\Delta}(\mathrm{c}) \Delta \mathrm{c}=\int_{w\left(\kappa_{1}\right)}^{w\left(\kappa_{2}\right)}\left(\Upsilon \circ w^{-1}\right)(s) \hat{\Delta} s
$$

or

$$
\int_{\kappa_{1}}^{\kappa_{2}} \Upsilon(\mathrm{c}) w^{\nabla}(\mathrm{c}) \nabla \mathrm{c}=\int_{w\left(\kappa_{1}\right)}^{w\left(\kappa_{2}\right)}\left(\Upsilon \circ w^{-1}\right)(s) \hat{\nabla} s
$$

Remark 2.1 Note that

$$
\int_{\kappa_{1}}^{\kappa_{2}} \Upsilon(\mathrm{c}) \Delta \mathrm{c}=\sum_{\mathrm{c}=\kappa_{1}}^{\kappa_{2}-1} \Upsilon(\mathrm{c}) \text { and } \int_{\kappa_{1}}^{\kappa_{2}} \Upsilon(\mathrm{c}) \nabla \mathrm{c}=\sum_{\mathrm{c}=\kappa_{1}+1}^{\kappa_{2}} \Upsilon(\mathrm{c}) \text {. }
$$

Theorem 2.2 (Dual time scale substitution rule [24]) Let $\top$ be a time scale and $\hat{\top}:=\{s \in$ $\mathbb{R} ;-s \in \top\}$. Let $w: \mathbb{Z} \rightarrow \mathbb{R}$ be strictly increasing and $\hat{\top}:=w(\top)$ be a time scale. If $\Upsilon: \mathbb{Z} \rightarrow \mathbb{R}$ is a continuous function and $w$ is differentiable with $r d$-continuous derivative, then for $\kappa_{1}, \kappa_{2} \in T_{\left[\kappa_{1}, \kappa_{2}\right]}$ we have

$$
\int_{\kappa_{1}}^{\kappa_{2}} \Upsilon(\mathrm{c})\left(-w^{\Delta}\right)(\mathrm{c}) \Delta \mathrm{c}=\int_{w\left(\kappa_{2}\right)}^{w\left(\kappa_{1}\right)}\left(\Upsilon \circ w^{-1}\right)(s) \hat{\nabla} s
$$

or

$$
\int_{\kappa_{1}}^{\kappa_{2}} \Upsilon(\mathrm{c})\left(-w^{\nabla}\right)(\mathrm{c}) \nabla \mathrm{c}=\int_{w\left(\kappa_{2}\right)}^{w\left(\kappa_{1}\right)}\left(\Upsilon \circ w^{-1}\right)(s) \hat{\Delta} s
$$

The first result starts from the following main theorem.

Theorem 2.3 Let $\Upsilon: \mathbb{Z} \rightarrow \mathbb{R}$ be a convex function on $\left[\kappa_{1}, \kappa_{2}\right]_{\mathbb{Z}}$ and $\kappa_{1}, \kappa_{2} \in \mathbb{Z}$ with $\kappa_{1}<\kappa_{2}$. If $\kappa_{1}+\kappa_{2}$ is an even number, then we have

$$
\Upsilon\left(\frac{\kappa_{1}+\kappa_{2}}{2}\right) \leq \frac{2}{\kappa_{2}-\kappa_{1}}\left[\int_{\frac{\kappa_{1}+\kappa_{2}}{2}}^{\kappa_{2}} \Upsilon(\mathrm{c}) \Delta \mathrm{c}+\int_{\frac{\kappa_{1}+\kappa_{2}}{2}}^{\kappa_{2}} \Upsilon(\mathrm{c}) \nabla \mathrm{c}\right] \leq \Upsilon\left(\kappa_{1}\right)+\Upsilon\left(\kappa_{2}\right) .
$$

Proof Let $\mathrm{c} \in \mathrm{T}_{\left[\frac{\kappa_{1}+\kappa_{2}}{2}, \kappa_{2}\right]} \backslash\{0,1\}$ be fixed. Then we can see that

$$
z_{1}=\frac{2-\mathrm{c}}{2} \kappa_{1}+\frac{\mathrm{c}}{2} \kappa_{2}, \quad z_{2}=\frac{\mathrm{c}}{2} \kappa_{1}+\frac{2-\mathrm{c}}{2} \kappa_{2}
$$

are in $\left[\kappa_{1}, \kappa_{2}\right]_{\mathbb{Z}}$ and $z_{1}+z_{2}=\kappa_{1}+\kappa_{2}$ is even. Since $\frac{1}{2} \in T_{\left[z_{1}, z_{2}\right]}$ (or $\top_{\left[z_{2}, z_{1}\right]}$ ) and $\Upsilon$ is convex on $\left[z_{1}, z_{2}\right]_{\mathbb{Z}}$ (or $\left.\left[z_{2}, z_{1}\right]_{\mathbb{Z}}\right)$, we can deduce

$$
\begin{aligned}
\Upsilon\left(\frac{\kappa_{1}+\kappa_{2}}{2}\right) & =\Upsilon\left(\frac{z_{1}+z_{2}}{2}\right) \leq \frac{\Upsilon\left(z_{1}\right)+\Upsilon\left(z_{2}\right)}{2} \\
& =\frac{1}{2}\left[\Upsilon\left(\frac{\mathrm{c}}{2} \kappa_{1}+\frac{2-\mathrm{c}}{2} \kappa_{2}\right)+\Upsilon\left(\frac{2-\mathrm{c}}{2} \kappa_{1}+\frac{\mathrm{c}}{2} \kappa_{2}\right)\right] .
\end{aligned}
$$


Integrating both sides over $\top_{\left[\frac{\kappa_{1}+\kappa_{2}}{2}, \kappa_{2}\right]}$ we get

$$
\begin{aligned}
& \int_{T_{\left[\frac{\kappa_{1}+\kappa_{2}}{2}, \kappa_{2}\right]}} \Upsilon\left(\frac{\kappa_{1}+\kappa_{2}}{2}\right) \hat{\Delta} \mathrm{c} \\
& \leq \frac{1}{2}\left[\int_{\top_{\left[\frac{\kappa_{1}+\kappa_{2}}{2}, \kappa_{2}\right]}} \Upsilon\left(\frac{2-\mathrm{c}}{2} \kappa_{1}+\frac{\mathrm{c}}{2} \kappa_{2}\right) \hat{\Delta} \mathrm{c}\right. \\
& \left.+\int_{T_{\left[\frac{\kappa_{1}+\kappa_{2}}{2}, \kappa_{2}\right]}} \Upsilon\left(\frac{\mathrm{c}}{2} \kappa_{1}+\frac{2-\mathrm{c}}{2} \kappa_{2}\right) \hat{\Delta} \mathrm{c}\right]=\frac{1}{2}\left[h_{1}+h_{2}\right],
\end{aligned}
$$

where $\hat{\Delta}$ is the derivative operator on the time scale $\top_{\left[\frac{\kappa_{1}+\kappa_{2}}{2}, \kappa_{2}\right]}$.

Making use of Theorem 2.1 with $w(\mathrm{c}):=\frac{2\left(\mathrm{c}-\kappa_{1}\right)}{\kappa_{2}-\kappa_{1}}$ we get

$$
\begin{aligned}
h_{1} & =\int_{\top_{\left[\frac{\kappa_{1}+\kappa_{2}}{2}, \kappa_{2}\right]}} \Upsilon\left(\frac{2-\mathrm{c}}{2} \kappa_{1}+\frac{\mathrm{c}}{2} \kappa_{2}\right) \hat{\Delta} \mathrm{c} \\
& =\int_{1}^{2} \Upsilon\left(\frac{2-\mathrm{c}}{2} \kappa_{1}+\frac{\mathrm{c}}{2} \kappa_{2}\right) \hat{\Delta} \mathrm{c}=\frac{2}{\kappa_{2}-\kappa_{1}} \int_{\frac{\kappa_{1}+\kappa_{2}}{2}}^{\kappa_{2}} \Upsilon(\mathrm{c}) \Delta \mathrm{c},
\end{aligned}
$$

where we used that $w^{\Delta}(\mathrm{c})=\frac{2}{\kappa_{2}-\kappa_{1}}$ and $w\left(\left[\frac{\kappa_{1}+\kappa_{2}}{2}, \kappa_{2}\right]_{\mathbb{Z}}\right)=\top_{\left[\kappa_{1}, \frac{\kappa_{1}+\kappa_{2}}{2}\right]}$ is also a time scale.

On the other hand, for $w(\mathrm{c}):=\frac{2\left(\kappa_{2}-\mathrm{c}\right)}{\kappa_{2}-\kappa_{1}}$, we have

$$
\begin{aligned}
h_{2} & =\int_{\top_{\left[\frac{\kappa_{1}+\kappa_{2}}{2}, \kappa_{2}\right]}} \Upsilon\left(\frac{\mathrm{c}}{2} \kappa_{1}+\frac{2-\mathrm{c}}{2} \kappa_{2}\right) \hat{\Delta} \mathrm{c}=\int_{\top_{\left[\frac{\kappa_{1}+\kappa_{2}}{2}, \kappa_{2}\right]}}\left(\Upsilon \circ w^{-1}\right)(\mathrm{c}) \hat{\Delta} \mathrm{c} \\
& =\int_{0}^{1}\left(\Upsilon \circ w^{-1}\right)(\mathrm{c}) \hat{\Delta} \mathrm{c}=\int_{-1}^{0}\left(\Upsilon \circ w^{-1}\right)^{*}(s) \hat{\nabla} s \\
& =\int_{-1=\left(f^{-1} \circ w\right)\left(\frac{\kappa_{1}+\kappa_{2}}{2}\right)}^{0=\left(f^{-1} \circ w\right)\left(\kappa_{2}\right)} \Upsilon\left(\left(f^{-1} \circ w\right)^{-1}\right)(s) \hat{\nabla} s,
\end{aligned}
$$

where $f(s)=-s$ and we used that

$$
\left(\Upsilon \circ w^{-1}\right)^{*}(s)=\left(\Upsilon \circ w^{-1}\right)(-s)=\Upsilon\left(w^{-1}(-s)\right)=\Upsilon\left(\left(w^{-1} \circ f\right)(s)\right)=\Upsilon\left(\left(f^{-1} \circ w\right)^{-1}\right)(s)
$$

Since

$$
\left(f^{-1} \circ w\right)(s)=f^{-1}(w(s))=f^{-1}\left(\frac{2\left(\kappa_{2}-s\right)}{\kappa_{2}-\kappa_{1}}\right)=\frac{2\left(s-\kappa_{2}\right)}{\kappa_{2}-\kappa_{1}}
$$

we have $\left(f^{-1} \circ w\right)^{\Delta}(s)=\frac{2}{\kappa_{2}-\kappa_{1}}>0$ and hence $f^{-1} \circ w$ is strictly increasing. Thus, by making use of Theorem 2.2, we get

$$
h_{2}=\int_{-1}^{0} \Upsilon\left(f^{-1} \circ w\right)^{-1}(\mathrm{c}) \hat{\nabla} \mathrm{c}=\frac{2}{\kappa_{2}-\kappa_{1}} \int_{\frac{\kappa_{1}+\kappa_{2}}{2}}^{\kappa_{2}} \Upsilon(\mathrm{c}) \nabla \mathrm{c} .
$$

Thus, the one half of the inequality in (2.1) follows from (2.2)-(2.4). 
To prove the other half of the inequality in (2.1), we use the convexity of $\Upsilon$ and the following inequalities:

$$
\begin{aligned}
& \Upsilon\left(\frac{2-\mathrm{c}}{2} \kappa_{1}+\frac{\mathrm{c}}{2} \kappa_{2}\right) \leq \frac{2-\mathrm{c}}{2} \Upsilon\left(\kappa_{1}\right)+\frac{\mathrm{c}}{2} \Upsilon\left(\kappa_{2}\right) \\
& \Upsilon\left(\frac{\mathrm{c}}{2} \kappa_{1}+\frac{2-\mathrm{c}}{2} \kappa_{2}\right) \leq \frac{\mathrm{c}}{2} \Upsilon\left(\kappa_{1}\right)+\frac{2-\mathrm{c}}{2} \Upsilon\left(\kappa_{2}\right) .
\end{aligned}
$$

Adding these we obtain

$$
\Upsilon\left(\frac{2-\mathrm{c}}{2} \kappa_{1}+\frac{\mathrm{c}}{2} \kappa_{2}\right)+\Upsilon\left(\frac{\mathrm{c}}{2} \kappa_{1}+\frac{2-\mathrm{c}}{2} \kappa_{2}\right) \leq \Upsilon\left(\kappa_{1}\right)+\Upsilon\left(\kappa_{2}\right) .
$$

Integrating both sides over $\top_{\left[\frac{\kappa_{1}+\kappa_{2}}{2}, \kappa_{2}\right]}$ we get

$$
\begin{aligned}
& \int_{T_{\left[\frac{\kappa_{1}+\kappa_{2}}{2}, \kappa_{2}\right]}} \Upsilon\left(\frac{2-\mathrm{c}}{2} \kappa_{1}+\frac{\mathrm{c}}{2} \kappa_{2}\right) \hat{\Delta} \mathrm{c}+\int_{\mathrm{T}_{\left[\frac{\kappa_{1}+\kappa_{2}}{2}, \kappa_{2}\right]}} \Upsilon\left(\frac{\mathrm{c}}{2} \kappa_{1}+\frac{2-\mathrm{c}}{2} \kappa_{2}\right) \hat{\Delta} \mathrm{c} \\
& \quad \leq \Upsilon\left(\kappa_{1}\right)+\Upsilon\left(\kappa_{2}\right) .
\end{aligned}
$$

We can use the same method used above we get

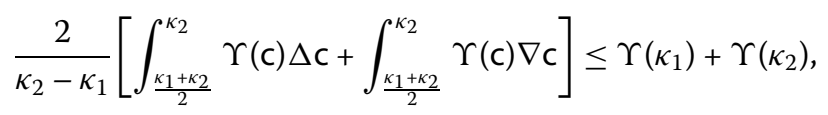

and thus the result follows.

\section{Discrete fractional inequality of HH-type}

The left nabla fractional sum of $\Upsilon$ of order $\varepsilon$ is defined by [25-27]

$$
\left({ }_{\kappa_{1}} \nabla^{-\varepsilon} \Upsilon\right)(\mathrm{c}):=\frac{1}{\Gamma(\varepsilon)} \sum_{r=\kappa_{1}+1}^{\mathrm{c}}(\mathrm{c}-\rho(r))^{\overline{\varepsilon-1}} \Upsilon(r)
$$

and the right delta fractional sum of $\Upsilon$ of order $\varepsilon$ is defined by $[25,26]$

$$
\left(\Delta_{\kappa_{2}}^{-\varepsilon} \Upsilon\right)(\mathrm{c}):=\frac{1}{\Gamma(\varepsilon)} \sum_{r=c+\varepsilon}^{\kappa_{2}}(r-\sigma(\mathrm{c}))^{(\varepsilon-1)} \Upsilon(r)
$$

for $\varepsilon \in \mathbb{R} \backslash\{\cdots,-2,-1,0\}$. For arbitrary $c, \varepsilon \in \mathbb{R}$ and $h>0$, the rising and falling factorial functions are, respectively, defined by [27]

$$
\begin{aligned}
& \mathrm{c}^{\bar{\varepsilon}}=\frac{\Gamma(\mathrm{c}+\varepsilon)}{\Gamma(\mathrm{c})}, \quad \mathrm{c} \in \mathbb{R} \backslash\{\ldots,-2,-1,0\}, \\
& \mathrm{c}^{(\varepsilon)}=\frac{\Gamma(\mathrm{c}+1)}{\Gamma(\mathrm{c}+1-\varepsilon)},
\end{aligned}
$$

such that $0^{\bar{\varepsilon}}=0$ and we use the convention that divisions at poles yield zero. 
Remark 3.1 In view of the rising and falling factorial functions in (3.3), we have

$$
\left(\kappa_{2}-r+\varepsilon-1\right)^{(\varepsilon-1)}=\left(\kappa_{2}-r+1\right)^{\overline{\varepsilon-1}} .
$$

Theorem 3.1 Let $\Upsilon: \mathbb{Z} \rightarrow \mathbb{R}$ be a convex function on $\left[\kappa_{1}, \kappa_{2}\right]_{\mathbb{Z}}$ and $\kappa_{1}, \kappa_{2} \in \mathbb{Z}$ with $\kappa_{1}<\kappa_{2}$. If $\kappa_{1}+\kappa_{2}$ is an even number, then, for $\varepsilon>0$, we have

$$
\begin{aligned}
\Upsilon\left(\frac{\kappa_{1}+\kappa_{2}}{2}\right) & \leq \frac{\Gamma(\varepsilon)}{\Omega\left(\kappa_{2}-\kappa_{1}\right)}\left[\left(\Delta_{\kappa_{2}-1}^{-\varepsilon} \Upsilon\right)\left(\frac{\kappa_{1}+\kappa_{2}}{2}-\varepsilon\right)+\left(\frac{\kappa_{1}+\kappa_{2}}{2} \nabla^{-\varepsilon} \Upsilon\right)\left(\kappa_{2}\right)\right] \\
& \leq \Upsilon\left(\kappa_{1}\right)+\Upsilon\left(\kappa_{2}\right),
\end{aligned}
$$

where

$$
\Omega=\int_{\top_{\left[\frac{\kappa_{1}+\kappa_{2}}{2}, \kappa_{2}\right]}}\left(\frac{\kappa_{2}-\kappa_{1}}{2}(\mathrm{c}-1)+(\varepsilon-1)\right)^{\overline{\varepsilon-1}} \hat{\Delta} \mathrm{c} .
$$

Proof Let $c \in T_{\left[\frac{\kappa_{1}+\kappa_{2}}{2}, \kappa_{2}\right]}$. Then we can see that

$$
z_{1}=\frac{\mathrm{c}}{2} \kappa_{1}+\frac{2-\mathrm{c}}{2} \kappa_{2}, \quad z_{2}=\frac{2-\mathrm{c}}{2} \kappa_{1}+\frac{\mathrm{c}}{2} \kappa_{2}
$$

are in $\left[\kappa_{1}, \kappa_{2}\right]_{\mathbb{Z}}$ and $z_{1}+z_{2}=\kappa_{1}+\kappa_{2}$ is even. Since $\frac{1}{2} \in\left[z_{1}, z_{2}\right]_{\mathbb{Z}}$ (or $\left[z_{2}, z_{1}\right]_{\mathbb{Z}}$ ) and $\Upsilon$ is convex on $\left[z_{1}, z_{2}\right]_{\mathbb{Z}}$ (or $\left.\left[z_{2}, z_{1}\right]_{\mathbb{Z}}\right)$, we can deduce

$$
\begin{aligned}
\Upsilon\left(\frac{\kappa_{1}+\kappa_{2}}{2}\right) & =\Upsilon\left(\frac{z_{1}+z_{2}}{2}\right) \leq \frac{\Upsilon\left(z_{1}\right)+\Upsilon\left(z_{2}\right)}{2} \\
& =\frac{1}{2}\left[\Upsilon\left(\frac{\mathrm{c}}{2} \kappa_{1}+\frac{2-\mathrm{c}}{2} \kappa_{2}\right)+\Upsilon\left(\frac{2-\mathrm{c}}{2} \kappa_{1}+\frac{\mathrm{c}}{2} \kappa_{2}\right)\right]
\end{aligned}
$$

Multiplying both sides by $\left(\frac{\kappa_{2}-\kappa_{1}}{2}(c-1)+(\varepsilon-1)\right)^{(\varepsilon-1)}$ and then integrating over $\top_{\left[\frac{\kappa_{1}+\kappa_{2}}{2}, \kappa_{2}\right]}$ we get

$$
\begin{aligned}
\Upsilon( & \left.\frac{\kappa_{1}+\kappa_{2}}{2}\right) \int_{\top_{\left[\frac{\kappa_{1}+\kappa_{2}}{2}, \kappa_{2}\right]}}\left(\frac{\kappa_{2}-\kappa_{1}}{2}(\mathrm{c}-1)+(\varepsilon-1)\right)^{(\varepsilon-1)} \hat{\Delta} \mathrm{c} \\
\leq & \frac{1}{2}\left[\int_{\top_{\left[\frac{\kappa_{1}+\kappa_{2}}{2}, \kappa_{2}\right]}}\left(\frac{\kappa_{2}-\kappa_{1}}{2}(\mathrm{c}-1)+(\varepsilon-1)\right)^{(\varepsilon-1)} \Upsilon\left(\frac{2-\mathrm{c}}{2} \kappa_{1}+\frac{\mathrm{c}}{2} \kappa_{2}\right) \hat{\Delta} \mathrm{c}\right. \\
& \left.+\int_{\top_{\left[\frac{\kappa_{1}+\kappa_{2}}{2}, \kappa_{2}\right]}}\left(\frac{\kappa_{2}-\kappa_{1}}{2}(\mathrm{c}-1)+(\varepsilon-1)\right)^{(\varepsilon-1)} \Upsilon\left(\frac{\mathrm{c}}{2} \kappa_{1}+\frac{2-\mathrm{c}}{2} \kappa_{2}\right) \hat{\Delta} \mathrm{c}\right] \\
:= & \frac{1}{2}\left[h_{1}+h_{2}\right],
\end{aligned}
$$

where $\hat{\Delta}$ is the derivative operator on the time scale $\top_{\left[\frac{\kappa_{1}+\kappa_{2}}{2}, \kappa_{2}\right]}$.

We assert that

$$
h_{1}:=\frac{2 \Gamma(\varepsilon)}{\kappa_{2}-\kappa_{1}}\left(\Delta_{\kappa_{2}-1}^{-\varepsilon} \Upsilon\right)\left(\frac{\kappa_{1}+\kappa_{2}}{2}-\varepsilon\right) \text {. }
$$


To prove this, we define $w(\mathrm{c}):=\frac{2\left(\mathrm{c}-\kappa_{1}\right)}{\kappa_{2}-\kappa_{1}}, g(\mathrm{c}):=\left(\mathrm{c}-\frac{\kappa_{1}+\kappa_{2}}{2}+(\varepsilon-1)\right)^{\overline{\varepsilon-1}}$ and $F(\mathrm{c})=g(\mathrm{c}) \Upsilon(\mathrm{c})$, then we have

$$
\begin{aligned}
F\left(w^{-1}(\mathrm{c})\right) & =(g f)\left(w^{-1}(\mathrm{c})\right)=g\left(w^{-1}(\mathrm{c})\right) \Upsilon\left(w^{-1}(\mathrm{c})\right) \\
& =\left(\frac{\kappa_{2}-\kappa_{1}}{2}(\mathrm{c}-1)+(\varepsilon-1)\right)^{(\varepsilon-1)} \Upsilon\left(\frac{2-\mathrm{c}}{2} \kappa_{1}+\frac{\mathrm{c}}{2} \kappa_{2}\right) .
\end{aligned}
$$

Then, by making use of Theorem 2.1 for the above findings, we get

$$
\begin{aligned}
h_{1} & =\int_{T_{\left[\frac{\kappa_{1}+\kappa_{2}}{2}, \kappa_{2}\right]}}\left(\frac{\kappa_{2}-\kappa_{1}}{2}(\mathrm{c}-1)+(\varepsilon-1)\right)^{(\varepsilon-1)} \Upsilon\left(\frac{2-\mathrm{c}}{2} \kappa_{1}+\frac{\mathrm{c}}{2} \kappa_{2}\right) \hat{\Delta} \mathrm{c} \\
& =\int_{\frac{\kappa_{1}+\kappa_{2}}{2}}^{\kappa_{2}} F(\mathrm{c}) w^{\Delta}(\mathrm{c}) \Delta \mathrm{c}=\frac{2}{\kappa_{2}-\kappa_{1}} \sum_{r=\frac{\kappa_{1}+\kappa_{2}}{2}}^{\kappa_{2}-1}\left(r-\frac{\kappa_{1}+\kappa_{2}}{2}+(\varepsilon-1)\right)^{(\varepsilon-1)} \Upsilon(r) \\
& =\frac{2 \Gamma(\varepsilon)}{\kappa_{2}-\kappa_{1}}\left(\Delta_{\kappa_{2}-1}^{-\varepsilon} \Upsilon\right)\left(\frac{\kappa_{1}+\kappa_{2}}{2}-\varepsilon\right) .
\end{aligned}
$$

This completes the proof of our assertion.

On the other hand, we assert that

$$
h_{2}:=\left(\frac{\kappa_{1}+\kappa_{2}}{2} \nabla^{-\varepsilon} \Upsilon\right)\left(\kappa_{2}\right)
$$

Then, for $w(c):=\frac{2\left(\kappa_{2}-c\right)}{\kappa_{2}-\kappa_{1}}$, we have

$$
\begin{aligned}
h_{2} & =\int_{T_{\left[\frac{\kappa_{1}+\kappa_{2}}{2}, \kappa_{2}\right]}}\left(\frac{\kappa_{2}-\kappa_{1}}{2}(\mathrm{c}-1)+(\varepsilon-1)\right)^{(\varepsilon-1)} \Upsilon\left(\frac{\mathrm{c}}{2} \kappa_{1}+\frac{2-\mathrm{c}}{2} \kappa_{2}\right) \hat{\Delta} \mathrm{c} \\
& =\int_{T_{\left[\frac{\kappa_{1}+\kappa_{2}}{2}, \kappa_{2}\right]}}\left(F \circ w^{-1}\right)(\mathrm{c}) \hat{\Delta} \mathrm{c} \\
& =\int_{0}^{1}\left(F \circ w^{-1}\right)(\mathrm{c}) \hat{\Delta} \mathrm{c}=\int_{-1}^{0}\left(F \circ w^{-1}\right)^{*}(s) \hat{\nabla} s \\
& =\int_{-1=\left(f^{-1} \circ w\right)\left(\frac{\kappa_{1}+\kappa_{2}}{2}\right)}^{0=\left(f^{-1} \circ w\right)\left(\kappa_{2}\right)} F\left(\left(f^{-1} \circ w\right)^{-1}\right)(s) \hat{\nabla} s,
\end{aligned}
$$

where $f(s)=-s, g(\mathrm{c}):=\left(\kappa_{2}-\mathrm{c}+(\varepsilon-1)\right)^{\overline{\varepsilon-1}}, F(\mathrm{c})=g(\mathrm{c}) \Upsilon(\mathrm{c})$ and we used that

$$
\left(F \circ w^{-1}\right)^{*}(s)=F\left(w^{-1}(-s)\right)=F\left(\left(w^{-1} \circ f\right)(s)\right)=F\left(\left(f^{-1} \circ w\right)^{-1}\right)(s) .
$$

Since

$$
\left(f^{-1} \circ w\right)(s)=f^{-1}(w(s))=f^{-1}\left(\frac{2\left(\kappa_{2}-s\right)}{\kappa_{2}-\kappa_{1}}\right)=\frac{2\left(s-\kappa_{2}\right)}{\kappa_{2}-\kappa_{1}}
$$

we have $\left(f^{-1} \circ w\right)^{\Delta}(s)=\frac{2}{\kappa_{2}-\kappa_{1}}>0$ and hence $f^{-1} \circ w$ is strictly increasing. Therefore, by making use of Theorem 2.2 and Remark 3.1, we get

$$
h_{2}=\int_{-1}^{0} F\left(f^{-1} \circ w\right)^{-1}(\mathrm{c}) \hat{\nabla} \mathrm{c}=\frac{2}{\kappa_{2}-\kappa_{1}} \int_{\frac{\kappa_{1}+\kappa_{2}}{2}}^{\kappa_{2}} F(\mathrm{c}) \nabla \mathrm{c}
$$




$$
\begin{aligned}
& =\frac{2}{\kappa_{2}-\kappa_{1}} \sum_{r=\frac{\kappa_{1}+\kappa_{2}}{2}+1}^{\kappa_{2}}\left(\kappa_{2}-r+\varepsilon-1\right)^{(\varepsilon-1)} \Upsilon(r) \\
& =\frac{2}{\kappa_{2}-\kappa_{1}} \sum_{r=\frac{\kappa_{1}+\kappa_{2}}{2}+1}^{\kappa_{2}}\left(\kappa_{2}-\sigma(r)\right)^{\overline{\varepsilon-1}} \Upsilon(r)=\frac{2 \Gamma(\varepsilon)}{\kappa_{2}-\kappa_{1}}\left(\frac{\kappa_{1}+\kappa_{2}}{2} \nabla^{-\varepsilon} \Upsilon\right)\left(\kappa_{2}\right) .
\end{aligned}
$$

This completes the second assertion and thus

$$
\Upsilon\left(\frac{\kappa_{1}+\kappa_{2}}{2}\right) \leq \frac{\Gamma(\varepsilon)}{\Omega\left(\kappa_{2}-\kappa_{1}\right)}\left[\left(\Delta_{\kappa_{2}-1}^{-\varepsilon} \Upsilon\right)\left(\frac{\kappa_{1}+\kappa_{2}}{2}-\varepsilon\right)+\left(\frac{\kappa_{1}+\kappa_{2}}{2} \nabla^{-\varepsilon} \Upsilon\right)\left(\kappa_{2}\right)\right]
$$

To prove the other half of the inequality in (2.1), we use the convexity of $\Upsilon$ and the following inequalities:

$$
\begin{aligned}
& \Upsilon\left(\frac{2-\mathrm{c}}{2} \kappa_{1}+\frac{\mathrm{c}}{2} \kappa_{2}\right) \leq \frac{2-\mathrm{c}}{2} \Upsilon\left(\kappa_{1}\right)+\frac{\mathrm{c}}{2} \Upsilon\left(\kappa_{2}\right) ; \\
& \Upsilon\left(\frac{\mathrm{c}}{2} \kappa_{1}+\frac{2-\mathrm{c}}{2} \kappa_{2}\right) \leq \frac{\mathrm{c}}{2} \Upsilon\left(\kappa_{1}\right)+\frac{2-\mathrm{c}}{2} \Upsilon\left(\kappa_{2}\right) .
\end{aligned}
$$

Adding these we obtain

$$
\Upsilon\left(\frac{c}{2} \kappa_{1}+\frac{2-c}{2} \kappa_{2}\right)+\Upsilon\left(\frac{2-c}{2} \kappa_{1}+\frac{c}{2} \kappa_{2}\right) \leq \Upsilon\left(\kappa_{1}\right)+\Upsilon\left(\kappa_{2}\right) .
$$

Multiplying both sides by $\left(\frac{\kappa_{2}-\kappa_{1}}{2}(c-1)+(\varepsilon-1)\right)^{(\varepsilon-1)}$ and then integrating both sides over $T_{\left[\frac{\kappa_{1}+\kappa_{2}}{2}, \kappa_{2}\right]}$ we get

$$
\begin{aligned}
& \int_{T_{\left[\frac{\kappa_{1}+\kappa_{2}}{2}, \kappa_{2}\right]}}\left(\frac{\kappa_{2}-\kappa_{1}}{2}(\mathrm{c}-1)+(\varepsilon-1)\right)^{(\varepsilon-1)} \Upsilon\left(\frac{\mathrm{c}}{2} \kappa_{1}+\frac{2-\mathrm{c}}{2} \kappa_{2}\right) \hat{\Delta} \mathrm{c} \\
& \quad+\int_{\mathrm{T}_{\left[\frac{\kappa_{1}+\kappa_{2}}{2}, \kappa_{2}\right]}}\left(\frac{\kappa_{2}-\kappa_{1}}{2}(\mathrm{c}-1)+(\varepsilon-1)\right)^{(\varepsilon-1)} \Upsilon\left(\frac{2-\mathrm{c}}{2} \kappa_{1}+\frac{\mathrm{c}}{2} \kappa_{2}\right) \hat{\Delta} \mathrm{c} \\
& \leq\left[\Upsilon\left(\kappa_{1}\right)+\Upsilon\left(\kappa_{2}\right)\right] \int_{\mathrm{T}_{\left[\frac{\kappa_{1}+\kappa_{2}}{2}, \kappa_{2}\right]}}\left(\frac{\kappa_{2}-\kappa_{1}}{2}(\mathrm{c}-1)+(\varepsilon-1)\right)^{(\varepsilon-1)} \hat{\Delta} \mathrm{c} .
\end{aligned}
$$

We can use the same method used above to get

$$
\frac{\Gamma(\varepsilon)}{\Omega\left(\kappa_{2}-\kappa_{1}\right)}\left[\left(\Delta_{\kappa_{2}-1}^{-\varepsilon} \Upsilon\right)\left(\frac{\kappa_{1}+\kappa_{2}}{2}-\varepsilon\right)+\left(\frac{\kappa_{1}+\kappa_{2}}{2} \nabla^{-\varepsilon} \Upsilon\right)\left(\kappa_{2}\right)\right] \leq \Upsilon\left(\kappa_{1}\right)+\Upsilon\left(\kappa_{2}\right)
$$

and thus the result follows.

Remark 3.2 In the literature of fractional integral inequalities there are three major HHtype inequalities, namely the endpoint, midpoint and end-midpoint $\mathrm{HH}$-types inequalities; for more details we advise the reader to read the Discussion section of Ref. [28]. Fortunately, the endpoint version of $\mathrm{HH}$-type inequality in the time scale notation has been established by Atıcı and Yaldız in [10]. Also, the inequality (3.4) obtained in Theorem 3.1 represents the midpoint version of $\mathrm{HH}$-type inequality which has never been presented before. 


\title{
4 Conclusion
}

The inequality of HH-type plays a crucial role in the theory and application of convex functions. During the last two decades, it has been used as an essential tool to obtain many results in approximation theory, integral inequalities, numerical analysis and optimization theory. In this study, we have considered new discrete time scales to obtain some inequalities of midpoint type for convex functions which have never presented before.

\author{
Acknowledgements \\ This research was funded by the Deanship of Scientific Research at Princess Nourah bint Abdulrahman University through \\ the Fast-track Research Funding Program. \\ Funding \\ Not applicable. \\ Availability of data and materials \\ Not applicable. \\ Competing interests \\ The authors declare that they have no competing interests. \\ Consent for publication \\ Not applicable.

\section{Authors' contributions} \\ All authors contributed equally and significantly in writing this article. All authors read and approved the final manuscript.

\section{Author details} \\ ${ }^{1}$ Department of Mathematics, College of Education, University of Sulaimani, Sulaimani, Kurdistan Region, Iraq. \\ ${ }^{2}$ Department of Mathematics and General Sciences, Prince Sultan University, P.O. Box 66833, Riyadh 11586, Saudi Arabia. \\ ${ }^{3}$ Department of Medical Research, China Medical University, Taichung 40402, Taiwan. ${ }^{4}$ Department of Computer Science \\ and Information Engineering, Asia University, Taichung, Taiwan. ${ }^{5}$ Department of Mathematical Sciences, Princess Nourah \\ bint Abdulrahman University, Riyadh, Saudi Arabia. ${ }^{6}$ Department of Mathematics, Cankaya University, 06790 Etimesgut, \\ Ankara, Turkey.
}

\section{Publisher's Note}

Springer Nature remains neutral with regard to jurisdictional claims in published maps and institutional affiliations.

Received: 28 July 2020 Accepted: 10 February 2021 Published online: 23 February 2021

References

1. Awan, M.U., Noor, M.A., Safdar, F., Islam, A., Mihai, M.V., Noor, K.I.: Hermite-Hadamard type inequalities with applications. Miskolc Math. Notes 21, 593-614 (2020)

2. Awan, M.U., Akhtar, N., Iftikhar, S., Noor, M.A., Chu, Y.-M.: New Hermite-Hadmard type inequalities for n-polynomial harmonically convex functions. J. Inequal. Appl. 2020, 125 (2020)

3. Mohammed, P.O.: Some new Hermite-Hadmard type inequalities for MT-convex functions on differentiable coordinates. J. King Saud Univ., Sci. 30, 258-262 (2018)

4. Latif, M.A., Rashid, S., Dragomir, S.S., Chu, Y.-M..: Hermite-Hadmard type inequalities for co-ordinated convex and qausi-convex functions and their applications. J. Inequal. Appl. 2019, 317 (2019)

5. Gürbüz, M., Akdemir, A.O., Rashid, S., Set, E.: Hermite-Hadamard type inequalities for co-ordinated convex and qausi-convex functions and their applications. J. Inequal. Appl. 2020, 172 (2020)

6. Sarikaya, M.Z., Set, E., Yaldiz, H., Başak, N.: Hermite-Hadamard's inequalities for fractional integrals and related fractional inequalities. Math. Comput. Model. 57, 2403-2407 (2013)

7. Sarikaya, M.Z., Yildirim, H.: On Hermite-Hadamard type inequalities for Riemann-Liouville fractional integrals. Miskolc Math. Notes 17(2), 1049-1059 (2017)

8. Anastassiou, G.A.: Nabla discrete fractional calculus and nabla inequalities. Math. Comput. Model. 51, 562-571 (2010)

9. Agarwal, R., O'Regan, D., Saker, S.: Dynamic Inequalities on Time Scales. Springer, New York (2014)

10. Atıcı, F.M., Yaldız, H.: Convex functions on discrete time domains. Can. Math. Bull. 59(2), 225-233 (2016)

11. Yaldız, H., Agarwal, P.: s-convex functions on discrete time domains. Analysis 37(4), 179-184 (2017)

12. Atıcl, F.M., Eloe, P.W.: Discrete fractional calculus with the Nabla operator. Electron. J. Qual. Theory Differ. Equ. 1, 3 (2009)

13. Mohammed, P.O.: Some integral inequalities of fractional quantum type. Malaya J. Mat. 4(1), 93-99 (2016)

14. Awan, M.U., Talib, S., Chu, Y.-M., Noor, M.A., Noor, K.I.: Some new refinements of Hermite-Hadamard-type inequalities involving $\psi_{k}$-Riemann-Liouville fractional integrals and applications. Math. Probl. Eng. 2020, 3051920 (2020)

15. Fernandez, A., Mohammed, P.: Hermite-Hadamard inequalities in fractional calculus defined using Mittag-Leffler kernels. Math. Methods Appl. Sci., 1-18 (2020). https://doi.org/10.1002/mma.6188

16. Mohammed, P.O.: Hermite-Hadamard inequalities for Riemann-Liouville fractional integrals of a convex function with respect to a monotone function. Math. Methods Appl. Sci., 1-11 (2019). https://doi.org/10.1002/mma.5784 
17. Mohammed, P.O., Abdeljawad, T.: Modification of certain fractional integral inequalities for convex functions. Adv. Differ. Equ. 2020, 69 (2020)

18. Mohammed, P.O., Brevik, I.: A new version of the Hermite-Hadamard inequality for Riemann-Liouville fractional integrals. Symmetry 12, 610 (2020). https://doi.org/10.3390/sym12040610

19. Rashid, S., Safdar, F., Akdemir, A.O., Noor, M.A., Noor, K.I.: Some new fractional integral inequalities for exponentially m-convex functions via extended generalized Mittag-Leffler function. J. Inequal. Appl. 2019, 299 (2019)

20. Zhou, S.-S., Rashid, S., Noor, M.A., Noor, K.I., Safdar, F., Chu, Y.-M.: New Hermite-Hadamard type inequalities for exponentially convex functions and applications. AIMS Math. 5(6), 6874-6901 (2020)

21. Mohammed, P.O., Sarikaya, M.Z.: On generalized fractional integral inequalities for twice differentiable convex functions. J. Comput. Appl. Math. 372, 112740 (2020)

22. Mohammed, P.O., Sarikaya, M.Z., Baleanu, D.: On the generalized Hermite-Hadamard inequalities via the tempered fractional integrals. Symmetry 12, 595 (2020). https://doi.org/10.3390/sym12040595

23. Huang, C.J., Rahman, G., Nisar, K.S., Ghaffar, A., Qi, F.: Some inequalities of Hermite-Hadamard type for k-fractional conformable integrals. Aust. J. Math. Anal. Appl. 16, 1-9 (2019)

24. Eloe, P.W., Sheng, Q., Henderson, J.: Notes on crossed symmetry solutions of the two-point boundary value problems on time scales. J. Differ. Equ. Appl. 9(1), 29-48 (2003)

25. Abdeljawad, T., Baleanu, D.: Monotonicity analysis of a nabla discrete fractional operator with discrete Mittag-Leffler kernel. Chaos Solitons Fractals 116, 1-5 (2017)

26. Abdeljawad, T., Baleanu, D.: Discrete fractional differences with nonsingular discrete Mittag-Leffler kernels. Adv. Differ. Equ. 2016, 232 (2016)

27. Goodrich, C., Peterson, A.: Discrete Fractional Calculus. Springer, Berlin (2015)

28. Mohammed, P.O., Abdeljawad, T., Kashuri, A.: Fractional Hermite-Hadamard-Fejer inequalities for a convex function with respect to an increasing function involving a positive weighted symmetric function. Symmetry 12(9), 1503 (2020)

\section{Submit your manuscript to a SpringerOpen ${ }^{\circ}$ journal and benefit from:}

- Convenient online submission

- Rigorous peer review

- Open access: articles freely available online

- High visibility within the field

- Retaining the copyright to your article

Submit your next manuscript at $\boldsymbol{s p r i n g e r o p e n . c o m ~}$ 